\title{
Insights into the association between coagulopathy and inflammation: abnormal clot mechanics are a warning of immunologic dysregulation following major injury
}

\author{
Stephanie A. Savage ${ }^{1}$, Ben L. Zarzaur ${ }^{1}$, Greg E. Gaski ${ }^{2}$, Tyler McCarroll ${ }^{3}$, Ruben Zamora ${ }^{4}$, \\ Rami A. Namas ${ }^{4}$, Yoram Vodovotz ${ }^{4}$, Rachael A. Callcut ${ }^{5}$, Timothy R. Billiar ${ }^{4}$, Todd O. McKinley ${ }^{3}$ \\ ${ }^{1}$ Department of Surgery, University of Wisconsin School of Medicine \& Public Health, Madison, Wisconsin, USA; ${ }^{2}$ Department of Orthopedics, \\ Inova Fairfax Medical Campus, Fairfax, Virginia, USA; ${ }^{3}$ Department of Orthopedics, Indiana University School of Medicine, Indianapolis, Indiana, \\ USA; ${ }^{4}$ Department of Surgery, University of Pittsburgh School of Medicine, Pittsburgh, Pennsylvania, USA; ${ }^{5}$ Department of Surgery, University of \\ California Davis School of Medicine, Davis, California, USA \\ Contributions: (I) Conception and design: TO McKinley, BL Zarzaur, SA Savage; (II) Administrative support: TO McKinley, GE Gaski; (III) \\ Provision of study materials or patients: Y Vodovotz, R Zamora, RA Namas, TR Billiar, TO McKinley; (IV) Collection and assembly of data: T \\ McCarroll, GE Gaski, SA Savage; (V) Data analysis and interpretation: SA Savage, BL Zarzaur, Y Vodovotz, RA Namas, R Zamora, TR Billiar, RA \\ Callcut, TO McKinley; (VI) Manuscript writing: All authors; (VII) Final approval of manuscript: All authors. \\ Correspondence to: Stephanie A. Savage, MD, MS. Department of Surgery, University of Wisconsin School of Medicine, G5/341 CSC, 600 Highland \\ Ave., Madison, WI 53792, USA. Email: savage@surgery.wisc.edu.
}

Background: Severe injury initiates a complex physiologic response encompassing multiple systems and varies phenotypically between patients. Trauma-induced coagulopathy may be an early warning of a poorly coordinated response at the molecular level, including a deleterious immunologic response and worsening of shock states. The onset of trauma-induced coagulopathy (TIC) may be subtle however. In previous work, we identified an early warning sign of coagulopathy from the admission thromboelastogram, called the MAR ratio. We hypothesized that a low MAR ratio would be associated with specific derangements in the inflammatory response.

Methods: In this prospective, observational study, 88 blunt trauma patients admitted to the intensive care unit (ICU) were identified. Concentrations of inflammatory mediators were recorded serially over the course of a week and the MAR ratio was calculated from the admission thromboelastogram. Correlation analysis was used to assess the relationship between MAR and inflammatory mediators. Dynamic network analysis was used to assess coordination of immunologic response.

Results: Seventy-nine percent of patients were male and mean age was 37 years (SD 12). The mean ISS was 30.2 (SD 12) and mortality was 7.2\%. CRITICAL patients (MAR ratio $\leq 14.2$ ) had statistically higher shock volumes at three time points in the first day compared to NORMAL patients (MAR ratio >14.2). CRITICAL patients had significant differences in IL-6 ( $\mathrm{P}=0.0065)$, IL-8 ( $\mathrm{P}=0.0115)$, IL-10 ( $\mathrm{P}=0.0316)$ and MCP-1 ( $\mathrm{P}=0.0039)$ concentrations compared to NORMAL. Differences in degree of expression and discoordination of immune response continued in CRITICAL patients throughout the first day.

Conclusions: The admission MAR ratio may be the earliest warning signal of a pathologic inflammatory response associated with hypoperfusion and TIC. A low MAR ratio is an early indication of complicated dysfunction of multiple molecular processes following trauma.

Keywords: Accidental injury; blood clotting; disseminated coagulation; intravascular; inflammation; thromboelastography

Submitted May 09, 2020. Accepted for publication Sep 18, 2020.

doi: 10.21037/atm-20-3651

View this article at: http://dx.doi.org/10.21037/atm-20-3651

(c) Annals of Translational Medicine. All rights reserved. 


\section{Introduction}

The Inflammation and Host Response to Injury research program, commonly known as the Glue Grant, has raised the awareness of the innate inflammatory response to injury since its inception in 1998 (1). This ongoing collaborative is one of the earliest research programs to elucidate the complex interplay between tissue damage, inflammation and a multitude of systemic responses. Defining an individual patient's physiologic response to severe injury is challenging, however. In addition to variability in type and degree of injury and the severity of shock at admission, there is likely a genotypic contribution that influences response at a molecular level $(2,3)$. In an age of personalized medicine, there is a focus on identifying biomarkers that reflect these underlying genotypic contributions to disease progression.

Activation of the inflammatory cascade following injury is meant to initiate healing, to prevent propagation of injury and to serve as a barrier to infection (4). The severity of the initial tissue insult contributes to the inflammatory response (5). In severe cases, trauma triggers a 'genomic storm,' in which dysconjugate expression of both proand anti-inflammatory pathways leads to a systemic inflammatory response syndrome (SIRS) $(6,7)$. SIRS develops in the days following injury, but the first inflammatory signature can be seen within $90 \mathrm{~min}$. of injury (8). The degree and duration of these inflammatory responses ultimately influences clinical course and outcome.

Inflammation and coagulation are known to interact at multiple points during their individual cascades, however. Inflammation may drive coagulation, coagulation may act to regulate the inflammatory cascade (9). In previous work, this group has evaluated the immediate coagulopathic response to injury, and how subtle early derangements in clot formation reflect a dysregulated immune response (10). The MAR ratio was created to incorporate specific aspects of the clotting cascade expressed from thromboelastography (TEG) - the MA reflecting clot strength and the R-time expressing onset of clot formation. By associating the onset of clot formation, including 'relative' delays, with the structural integrity of the subsequent clot formed, we postulated that there were significant malfunctions in clot formation present, despite seemingly normal individual TEG values. Accordingly, this ratio may be a sensitive biomarker of a patient-specific phenotype of abnormal clot formation.

The purpose of this study was to understand how early derangements in the coagulation system, quantified by the MAR ratio, reflect the magnitude and coordination of immune system activation. We hypothesized that low MAR ratios, pathologically associated with increased blood utilization and mortality, would be associated with specific early derangements in the immunologic response to injury when compared to patients with normal MAR ratios. We present the following article in accordance with the STROBE reporting checklist (available at http://dx.doi. org/10.21037/atm-20-3651).

\section{Methods}

This secondary analysis prospectively observed one hundred consecutive, multiply injured patients admitted to the trauma intensive care unit (TICU) or the operating room from April 2015 through September 2016. Patients were excluded if they had a non-survivable head injury, if they did not have an admission TEG, or if the TEG measurement was delayed, defined as more than $200 \mathrm{~min}$. following admission. Pregnant patients, those with a preexisting clotting disorder or actively taking antiplatelet or anticoagulant agents, those less than 18 years or greater than 60 years of age, and prisoners were excluded. The study was conducted in accordance with the Declaration of Helsinki (as revised in 2013). The study was approved by the Institutional Review Board of Indiana University School of Medicine (number 1411863767) and informed consent was taken from all individual participants.

Basic demographic data, injury characteristics, and laboratory data were collected. Total volumes of transfused packed red blood cells (PRBC), fresh frozen plasma (FFP), platelets, and cryoprecipitate (cryo) were recorded for the first 24 hours of admission. The primary outcome was the association between MAR ratio and inflammatory response. Secondary outcome variables included blood product utilization, as well as reaching critical administration threshold status (CAT +), a marker of large-volume hemorrhage defined as three units of PRBC transfused in any 60 minutes period (11). Intensive care unit (ICU) and hospital length of stay, and mortality were also recorded. There was no follow-up involved in this study.

\section{Calculation of the MAR ratio}

The MAR ratio was calculated from the admission TEG. The ratio was calculated as

MAR ratio $=\mathrm{MA} / \mathrm{R}$-time

Values less than or equal to 14.2 were considered 
abnormal (CRITICAL) based upon prior work (10). Values greater than 14.2 were considered NORMAL.

\section{Blood processing}

Blood was collected upon presentation to the Emergency Department (ED) (time 0), at 8 hours, 24 hours, and daily to day seven following injury or TICU discharge. Blood samples were all processed within two hours of collection. Samples were centrifuged at room temperature at 1,500 rpm for $10 \mathrm{~min}$. $1.0 \mathrm{~mL}$ of plasma was introduced into separate cryovial tubes and immediately frozen at -80 degrees Celsius.

\section{Evaluation of the inflammatory response to injury}

Serial quantification of inflammatory mediator values occurred over the first week following admission. An admission sample was taken immediately after arrival to the ED. Subsequent samples were taken at 8 and 24 hours and then daily until discharge from the TICU or day seven.

Plasma analyses were performed using a Luminex ${ }^{\mathrm{TM}}$ panel Bioassay of 20 inflammatory mediators for each patient. Mediators included Interleukin (IL)-10, IL-17A, IL-1 receptor antagonist (IL-1RA), IL-1 $\beta$, IL-4, IL-5, IL-6, IL-7, IL-8, interferon gamma-induced protein of $10 \mathrm{kDa}$ (IP-10), monocyte chemoattractant protein-1 (MCP-1), IL-22, IL-9, IL-33, IL-21, IL-23, IL-17E/IL-25, soluble IL-2 receptor- $\alpha$ (sIL-2R $\alpha$ ), and monokine induced by interferon gamma (MIG). High mobility group box 1 (HMGB1) was assayed using a specific enzyme-linked immunosorbent assay (ELISA-Shino Test Japan). A wide array of inflammatory mediators may be involved in the response to injury (12). Based on review of the literature, eight mediators were chosen a priori to evaluate the relationship between their expression and coagulopathy following trauma (IL-1 $\beta$, IL-4, IL-6, IL-8, IL-10, IL-23, HMGB1, and MCP-1) (4,6,7,13-18). Correlation analysis was used to define the relationship between the MAR ratio and these mediators. Mediators demonstrating a significant relationship to the MAR ratio were then further assessed using multivariable linear growth models, controlling for age and ISS.

Trauma-induced coagulopathy (TIC) is associated strongly with the magnitude of injury and hemorrhage (19). Therefore, propensity score matching was used to further refine the groups, by normalizing for injury severity and degree of shock. This allowed better identification of differences in immunologic responses between CRITICAL and NORMAL MAR ratio patients. CRITICAL patients were matched to NORMAL patients based on age, gender and ISS (INJURY subgroup). A second, more stringent matching (SHOCK subgroup) compared CRITICAL and NORMAL patients matched by age, gender, ISS and admission base deficit.

Dynamic Network Analysis (DyNA) was then used to demonstrate the degree of coordination in the immunologic response to injury over the first 24 hours $(20,21)$. This analysis was carried out to define, in a granular fashion, the central inflammatory network nodes as a function of both time and patient sub-group. Using inflammatory mediator measurements of at least three time-points for each patient, networks were created over two consecutive time periods $\left(0-8\right.$ and $8-24$ h) using MATLAB $^{\circledR}$ software $(10,11,15)$. Network edges (connections), defined as the number of trajectories of serum inflammatory mediators that move in parallel, were created if the Pearson correlation coefficient between any two nodes (inflammatory mediators) at the same time-interval was greater or equal to a threshold of 0.7 (a correlation value commonly used to characterize trajectories that move in parallel either up or down). The network complexity for each time-interval was calculated using the following formula: Sum $\left(\mathrm{N}_{1}+\mathrm{N}_{2}+\ldots+\mathrm{Nn}\right) / \mathrm{n}-1$, where $\mathrm{N}$ represents the number of connections for each mediator and $\mathrm{n}$ is the total number of mediators analyzed. The total number of network connections represents the sum of the number of connections across all time-intervals for all patients in a given patient sub-group.

\section{Statistical analysis}

Tests for normality were used and continuous variables were analyzed with either the $\chi^{2}$ or Kruskal-Wallis test. Correlation analysis was utilized to determine the relationship between the admission R-time and MA and the specified inflammatory mediators. Results were expressed as correlation coefficients with $\mathrm{P}$ values less than 0.05 demonstrating significance. Multivariable linear growth models, controlling for age and ISS, were used to compare the MAR ratio to significant inflammatory mediators. SAS v. 9.4 for Windows (SAS Institute, Cary, NC, USA) was utilized for these statistical analyses. A pairwise, retrospective 1:1 propensity matched sub-analysis was performed using IBM SPSS Statistics ${ }^{\circledR}$ case-control 
Table 1 Characteristics of CRITICAL and NORMAL patients

\begin{tabular}{|c|c|c|c|}
\hline Patient characteristics & CRITICAL (MAR <14.2) & NORMAL (MAR $\geq 14.2$ ) & $P$ value \\
\hline Gender (\% male) & $70 \%$ & $84 \%$ & 0.1966 \\
\hline Injury severity score (ISS) ${ }^{\dagger}$ & $30[22,41]$ & $27[17,36]$ & 0.1754 \\
\hline Mechanism of injury (\% blunt) & $100 \%$ & $96.5 \%$ & 0.3246 \\
\hline Shock index & $0.92(0.64,1.59)$ & $0.79(0.66,1.04)$ & 0.2374 \\
\hline Critical administration threshold (CAT+) (\%) & $52 \%$ & $23 \%$ & 0.0078 \\
\hline Red blood cells (units) ${ }^{\ddagger}$ & $11(17.5)$ & $2.8(5.9)$ & 0.0006 \\
\hline Fresh frozen plasma (units) ${ }^{\ddagger}$ & $4.8(8.4)$ & $1.2(3.1)$ & 0.0067 \\
\hline Hospital days $^{\dagger}$ & $8[6,25]$ & $15[8,20]$ & 0.2177 \\
\hline Intensive care unit (ICU), days ${ }^{\dagger}$ & $5[3,10]$ & $7[3,15]$ & 0.4154 \\
\hline Mortality (\%) & $7.4 \%$ & $7 \%$ & 0.3416 \\
\hline
\end{tabular}

${ }^{\dagger}$, interquartile ranges (confidence intervals); ${ }^{\ddagger}$, mean (standard deviation).

matching algorithm.

\section{Results}

One hundred patients were initially enrolled in this pilot study. Three patients with non-survivable brain injuries were excluded. Nine additional patients were removed due to missing or delayed TEG data. Eighty-eight patients were thus included in this analysis.

The mean age of the study population was 37 years (SD 12) and $79 \%$ of patients were male. Ninety-eight percent of patients suffered a blunt mechanism of injury. The mean ISS was 30.2 (SD 13). The mean hemoglobin at admission was $12.6 \mathrm{~g} / \mathrm{dL}$ (SD 2.4), median base excess was $-5.0 \mathrm{mEq} / \mathrm{L}(\mathrm{IQR},-7.5,-2.0)$ and median shock index was $0.84(0.66,1.09)$. The median ICU length of stay was 6 days (IQR, 3, 14) and the mean hospital length of stay was 15.5 days (SD 10.7). Overall mortality was $7.2 \%$.

The median MAR ratio for the entire cohort was 16.9 (IQR, 13.1, 20.0). Previous work has indicated that the R-time and MA may be the most important TEG values in predicting the propensity to develop TIC (10). For the 88 patients included in analysis, the median admission $\mathrm{R}$-time was 3.7 seconds (IQR, 3.1, 4.2). The median $\alpha$-angle was $70.9^{\circ}$ (IQR, 66.4, 73.6). Median admission MA was $61.4 \mathrm{~mm}$ (IQR, 55.4, 64.3). Median LY-30 was $0.005 \%$
(IQR, 0.001, 0.02).

Characteristics of CRITICAL and NORMAL patients are demonstrated in Table 1. There were no differences in patient age, gender, mechanism or injury or overall injury severity score. There was no statistical difference in ICU and hospital length of stay between groups, though NORMAL patients had a clinically longer ICU stay [7 days (IQR, 3, 15)] and hospital stay [15 days (IQR, 8, 20)], compared to CRITICAL patients [ICU 5 days (IQR 3, 10), $\mathrm{P}=0.4154$ and hospital 8 days (IQR, 6, 25), $\mathrm{P}=0.2177]$. Overall mortality was the same between groups.

Physiologic status at admission was evaluated with base deficit and shock index. CRITICAL patients had a significantly lower based deficit $[-7 \mathrm{mEq} / \mathrm{L}(-8,-2)]$ at the time of admission compared to NORMAL patients [-4 mEq/L $(-7,-2), \mathrm{P}=0.0081]$. Though the total shock index was much more significant in CRITICAL patients $[0.92(0.64,1.59)]$ than those in the NORMAL group $[0.8(0.66,1.04)]$, this did not reach statistical significance $(\mathrm{P}=0.2374)$.

CRITICAL patients required significantly more PRBC, FFP and platelets compared to NORMAL patients. Increased blood product utilization also translated to more CRITICAL patients reaching the critical administration threshold (CAT+). Fifty-two percent of CRITICAL patients 
Table 2 Cytokine Concentrations at Admission for CRITICAL and NORMAL subgroups

\begin{tabular}{lccc}
\hline Cytokines & CRITICAL (MAR <14.2) & NORMAL (MAR $\geq 14.2)$ & P value \\
\hline IL-1b (Interleukin 1 beta) & $1.09(0,2.6)$ & $1.32(0.3,2.8)$ & 0.3721 \\
IL-4 (Interleukin 4) & $0(0,0)$ & $0(0,0)$ & 0.6230 \\
IL-6 (Interleukin 6) & $302.5(72.7,1,145.0)$ & $94.0(43.7,272.4)$ & 0.0065 \\
IL-8 (Interleukin 8) & $75.6(23.7,117.8)$ & $24.2(12.7,67.6)$ & 0.0115 \\
IL-10 (Interleukin 10) & $140.1(79.4,463.0)$ & $83.8(28.1,226.1)$ & 0.0316 \\
IL-23 (Interleukin 23) & $9,644(5,487,20,802)$ & $15,474.5(5,441,32,000)$ & 0.2637 \\
MCP-1 (Monocyte Chemoattractant & $2,263(704,5,580)$ & $776.2(469.6,1,727)$ & 0.0039 \\
Protein 1) & $16.3(6.0,30.4)$ & $9.4(5.4,20.2)$ & 0.0970 \\
HMGB-1 (High Mobility Group Box 1) &
\end{tabular}

were CAT + compared to $23 \%$ of NORMAL patients $(\mathrm{P}=0.0078)$.

\section{Evaluation of cytokine response to injury}

Of the eight a priori inflammatory mediators, we compared the admission concentrations for CRITICAL and NORMAL patients (Table 2). CRITICAL patients had significantly higher concentrations of IL-6 [302.5 (IQR, 72.7, 1,145) vs. 94 (IQR, 43.7, 272.4), $\mathrm{P}=0.0065]$, IL-8 [75.6 (IQR, 23.7, 117.8) vs. 24.2 (IQR, 12.7, 67.6), $\mathrm{P}=0.0115]$, IL-10 [140.1 (IQR, 79.4, 463) vs. 83.8 (IQR, 28.1, 226.1), $\mathrm{P}=0.0316]$ and MCP-1 [2,263 (IQR, 704, 5,580) vs. 776.2 (IQR, 469.6, 1,727), $\mathrm{P}=0.0039]$. There were no differences in admission concentrations of IL-1b, IL-4, IL-23 or HMGB-1.

A correlation analysis was also performed between the eight a priori inflammatory mediators and the admission R-time and MA. A significant positive correlation was seen between the R-time and IL-6 (0.453, $\mathrm{P}<0.0001)$, IL-8 (0.466, $\mathrm{P}<0.0001)$ and MCP-1 (0.369, $\mathrm{P}=0.0003)$. A significant negative correlation was found between the MA and IL-6 (-0.387, $\mathrm{P}=0.0001)$, IL-8 (-0.310, $\mathrm{P}=0.0025)$, IL-10 (-0.261, $\mathrm{P}=0.0117)$ and MCP-1 (-0.300, $\mathrm{P}=0.0034)$.

\section{Subgroup analysis: concentrations of inflammatory mediators}

Propensity matching was performed based on age, gender and ISS (INJURY group, $\mathrm{N}=15$ per group), as well as for age, gender, ISS and admission base deficit (SHOCK Group, N=11 per group). For INJURY patients, concentrations of IL-10, IL-6, MCP-1 and HMBG-1 were significantly higher in CRITICAL patients than in NORMAL patients at 0hr. (Figure 1, NORMAL is represented by the vertical line at ' 1 '). CRITICAL patients also had significantly lower IL- $1 \mathrm{~b}$ at $0 \mathrm{~h}$. Concentrations of most cytokines equilibrated between CRITICAL and NORMAL over the first 24 hours in Group 1, except for IL- $1 \mathrm{~b}$ which was lower at $48 \mathrm{~h}$ in CRITICAL patients and IL-4 which remained significantly lower in CRITICAL patients at both 8 and $48 \mathrm{~h}$.

The SHOCK group, which accounted for admission physiology via inclusion of base deficit, resulted in 11 patients per matched group. IL-10 and HMBG-1 were elevated in CRITICAL patients, though this did not quite reach statistical significance $(0.05<\mathrm{P}<0.1)$ (Figure 2, NORMAL is represented by the vertical line at ' 1 '). By 48 hours from injury, however, CRITICAL patients in the SHOCK group did have a significantly lower IL-4 compared to NORMAL patients.

\section{Dynamic network analysis}

DyNA was used to characterize the coordination of the immune response following injury. CRITICAL patients in the INJURY subgroup had decreased coordination of their inflammatory mediators at the time of admission, with only 10 of 20 markers demonstrating connectivity with other mediators between 0 and $8 \mathrm{~h}$ after injury (Figure 3). Over the first day, the orchestration of the immune response became more robust, with more mediators involved and a greater degree of interconnectivity in CRITICAL patients from 8 to $24 \mathrm{~h}$. In comparison, NORMAL patients had 17 of 20 mediators with interconnectivity during the first $8 \mathrm{hr}$ after injury. This degree of coordination was perpetuated 


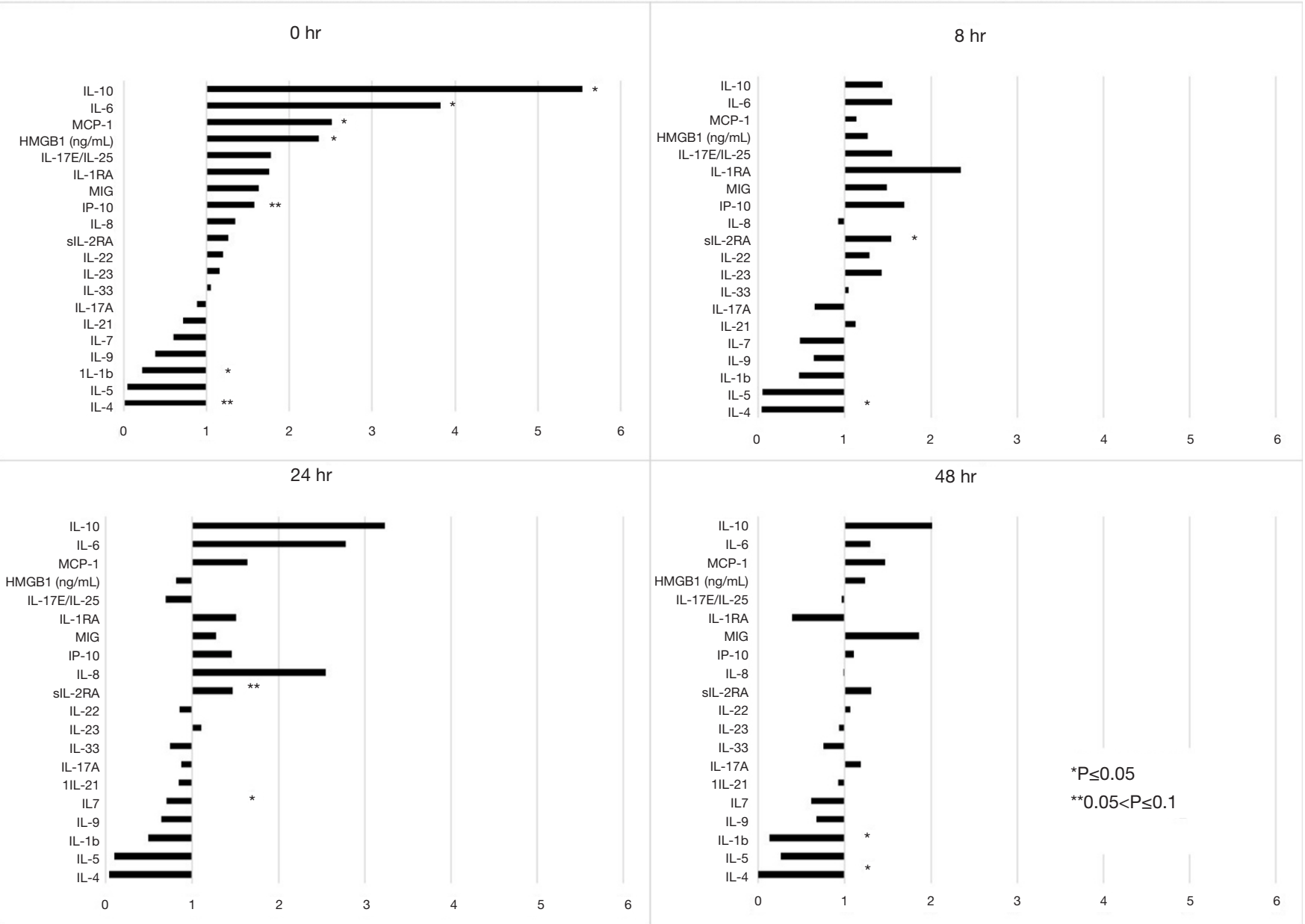

Figure 1 Expression of inflammatory mediators for CRITICAL and NORMAL patients in the Injury subgroup analysis at admission and 8 , 24 and 48 hours following admission. Cytokine expression is demonstrated for CRITICAL patients, in comparison to NORMAL patients, represented by the vertical line at '1'. For example, IL-10 in Group 1 CRITICAL patients, hour 0, is 5.5 fold greater than in NORMAL patients. IL-10, Interleukin 10; IL-6, Interleukin 6; MCP-1, Monocyte Chemoattractant Protein 1; HMGB-1, High Mobility Group Box 1; IL17E/IL-25, Interleukin 17E/Interleukin 25; IL-1RA, Interleukin 1 Receptor Antagonist; MIG, Monokine Induced by Gamma Interferon; IP-10, Interferon $\gamma$-induced Protein 10; IL-8, Interleukin 8; sIL-2RA, Soluble Interleukin 2 Receptor $\alpha$; IL-22, Interleukin 22; IL 23, Interleukin 23; IL-33, Interleukin 33; IL-17A, Interleukin 17A; IL-21, Interleukin 21; IL-7, Interleukin 7; IL-9, Interleukin 9; IL 1b, Interleukin 1 $\beta$; IL-5, Interleukin 5; IL 4, Interleukin 4.

over the first day. A similar pattern of coordination was seen with CRITICAL and NORMAL patients in the SHOCK subgroup (Figure 4).

\section{Conclusions}

Injury provokes a cascade of responses designed to control damage and return the organism to homeostasis. Both the coagulation and inflammatory cascades are stimulated by this process. Inter-connected, they propagate and regulate one another. Over-expression or malfunction in components of either system leads to maladaptive responses such as TIC and systemic inflammatory response syndrome (SIRS).

TIC is driven by multiple factors, including consumption of coagulation factors with unbalanced replacement, increased production of fibrinogen and factor VIII and increased activation of platelets $(15,22)$. Dysregulation of this system contributes to a feedback loop, which potentiates the shock state, increases the likelihood of multi-organ dysfunction (MOD) and includes abnormal 


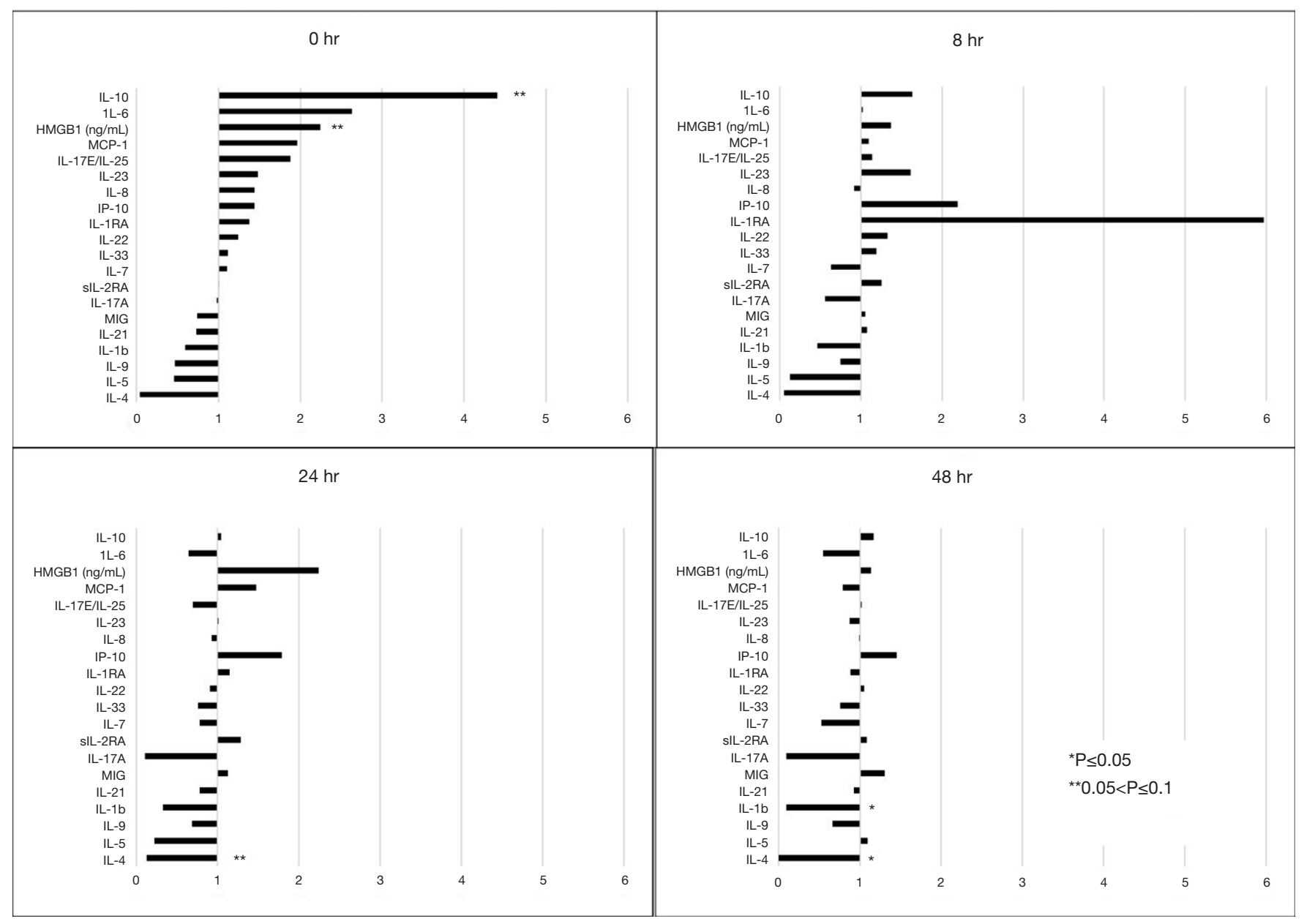

Figure 2 Expression of inflammatory mediators for CRITICAL and NORMAL patients in the Shock subgroup analysis at admission and 8, 24 and 48 hours following admission. Cytokine expression is demonstrated for CRITICAL patients, in comparison to NORMAL patients, represented by the vertical line at '1'. IL-10, Interleukin 10; IL-6, Interleukin 6; MCP-1, Monocyte Chemoattractant Protein 1; HMGB-1, High Mobility Group Box 1; IL17E/IL-25, Interleukin 17E/Interleukin 25; IL-1RA, Interleukin 1 Receptor Antagonist; MIG, Monokine Induced by Gamma Interferon; IP-10, Interferon $\gamma$-induced Protein 10; IL-8, Interleukin 8; sIL-2RA, Soluble Interleukin 2 Receptor $\alpha$; IL22, Interleukin 22; IL 23, Interleukin 23; IL-33, Interleukin 33; IL-17A, Interleukin 17A; IL-21, Interleukin 21; IL-7, Interleukin 7; IL-9, Interleukin 9; IL 1b, Interleukin 1 $\beta$; IL-5, Interleukin 5; IL 4, Interleukin 4.

inflammatory responses.

Patient-specific differences in the immunologic response to injury also contribute to TIC, as seen with the data from this study. In sub-cohorts matched for injury severity (INJURY) and further matched for injury severity and magnitude of shock (SHOCK), coagulopathic patients demonstrated a profound reduction in the coordination of their initial immunologic response, reflected by reduced connectivity in inflammatory mediators during the hours after injury. The poorly coordinated inflammatory response may lead to increased morbidity from hypoperfusion and shock states, as well as SIRS and MOD (13,22-25). Excess expression of pro-inflammatory cytokines and thrombin attract neutrophils, which are activated by the dysregulated inflammatory state, the 'genomic storm' $(7,14)$. Activated neutrophils also express myeloperoxidases and elastase, which contribute to the endothelial damage caused by the initial injury (14). Further, the injured endothelium then contributes to a pro-thrombotic, and typically antifibrinolytic, state. Via up-regulation of the inflammatory response, as well as sequelae of SIRS, injury leads to coagulopathy. 
DyNA - hours 0 to 8
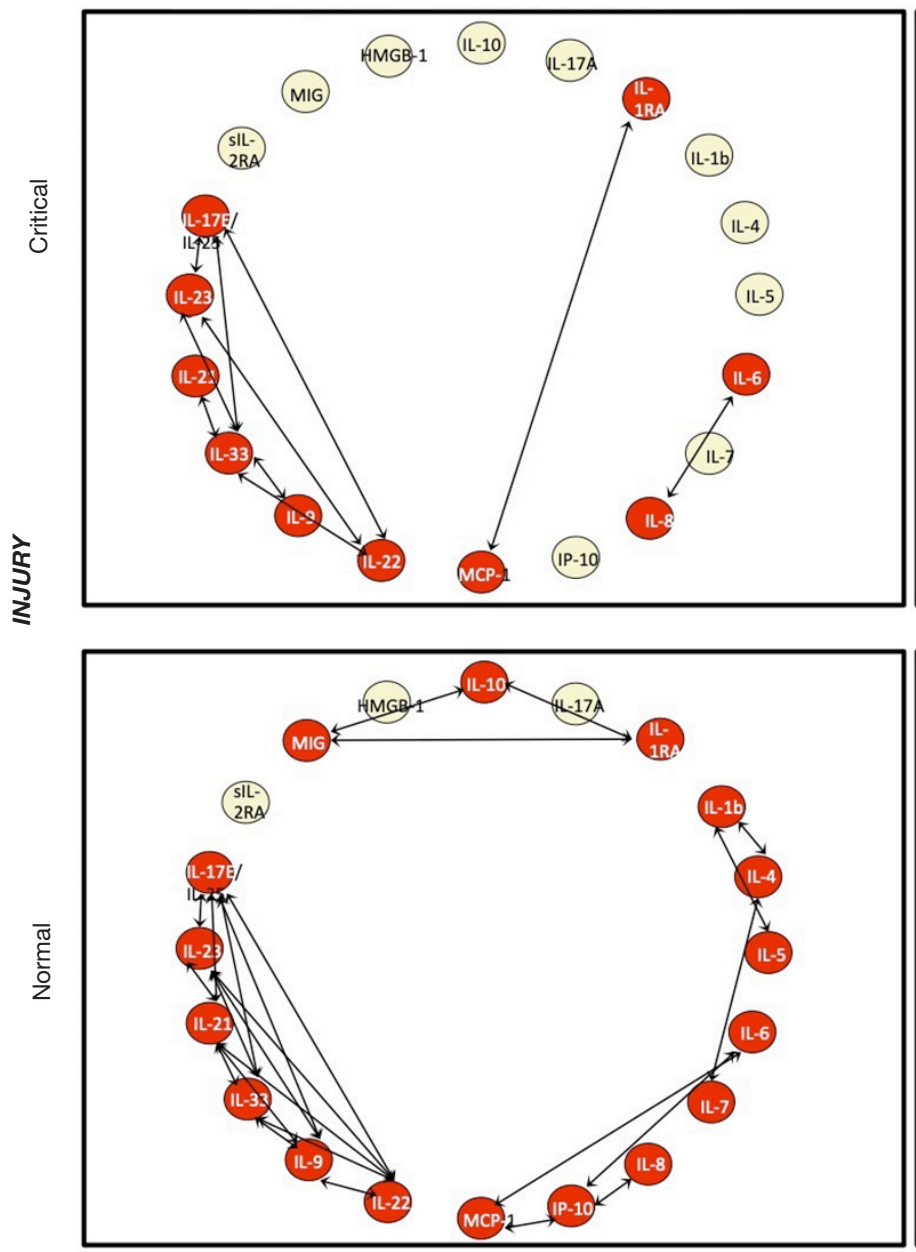

DyNA - hours 8 to 24
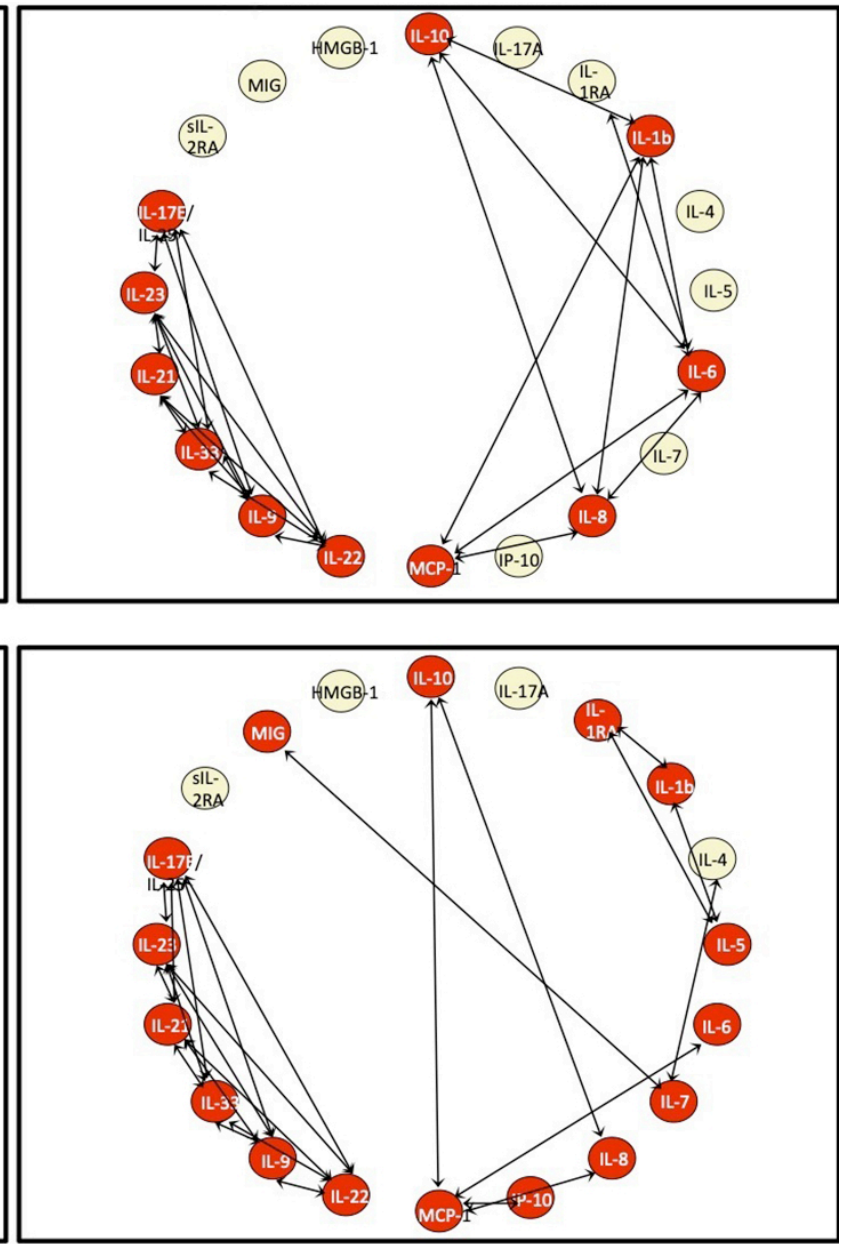

Figure 3 Dynamic network analysis (DyNA) demonstrating inflammatory coordination for the Injury subgroup analysis (matched for age, gender, and ISS). The top row represents CRITICAL patients at two time intervals (0-8 and 8-24 hours), while the bottom row represents normal patient over the same interval. Inflammatory mediators are nodes, with red nodes dynamically involved in interactions, represented by the arrows. DyNa, Dynamic Network Analysis; IL-10, Interleukin 10; IL-6, Interleukin 6; MCP-1, Monocyte Chemoattractant Protein 1; HMGB-1, High Mobility Group Box 1; IL17E/IL-25, Interleukin 17E/Interleukin 25; IL-1RA, Interleukin 1 Receptor Antagonist; MIG, Monokine Induced by Gamma Interferon; IP-10, Interferon $\gamma$-induced Protein 10; IL-8, Interleukin 8; sIL-2RA, Soluble Interleukin 2 Receptor $\alpha$; IL-22, Interleukin 22; IL 23, Interleukin 23; IL-33, Interleukin 33; IL-17A, Interleukin 17A; IL-21, Interleukin 21; IL-7, Interleukin 7; IL-9, Interleukin 9; IL 1b, Interleukin 1ß; IL-5, Interleukin 5; IL 4, Interleukin 4.

In the current study, we sought to determine if the low MAR ratio, which reflects the dysfunctional coagulation of TIC, also reflected the inflammatory milieu following major injury. Patients in this study were severely injured, as demonstrated by a mean ISS of 30 and a median admission base excess of $-5.0 \mathrm{mEq} / \mathrm{L}$. CRITICAL and NORMAL patients had similar injury severity scores, mechanism of injury, admission hemoglobin and admission shock index, though the shock index was quite clinically divergent.
CRITICAL patients did demonstrate a significantly worse base deficit. When focusing on transfusion requirements in these patients, CRITICAL patients required significantly more PRBC's, FFP and platelets. They also reached the critical administration threshold at double the rate of NORMAL patients. The fact that the two groups appear clinically similar at the moment of admission but demonstrate different blood product consumptions reaffirms the association between the MAR ratio and TIC. 


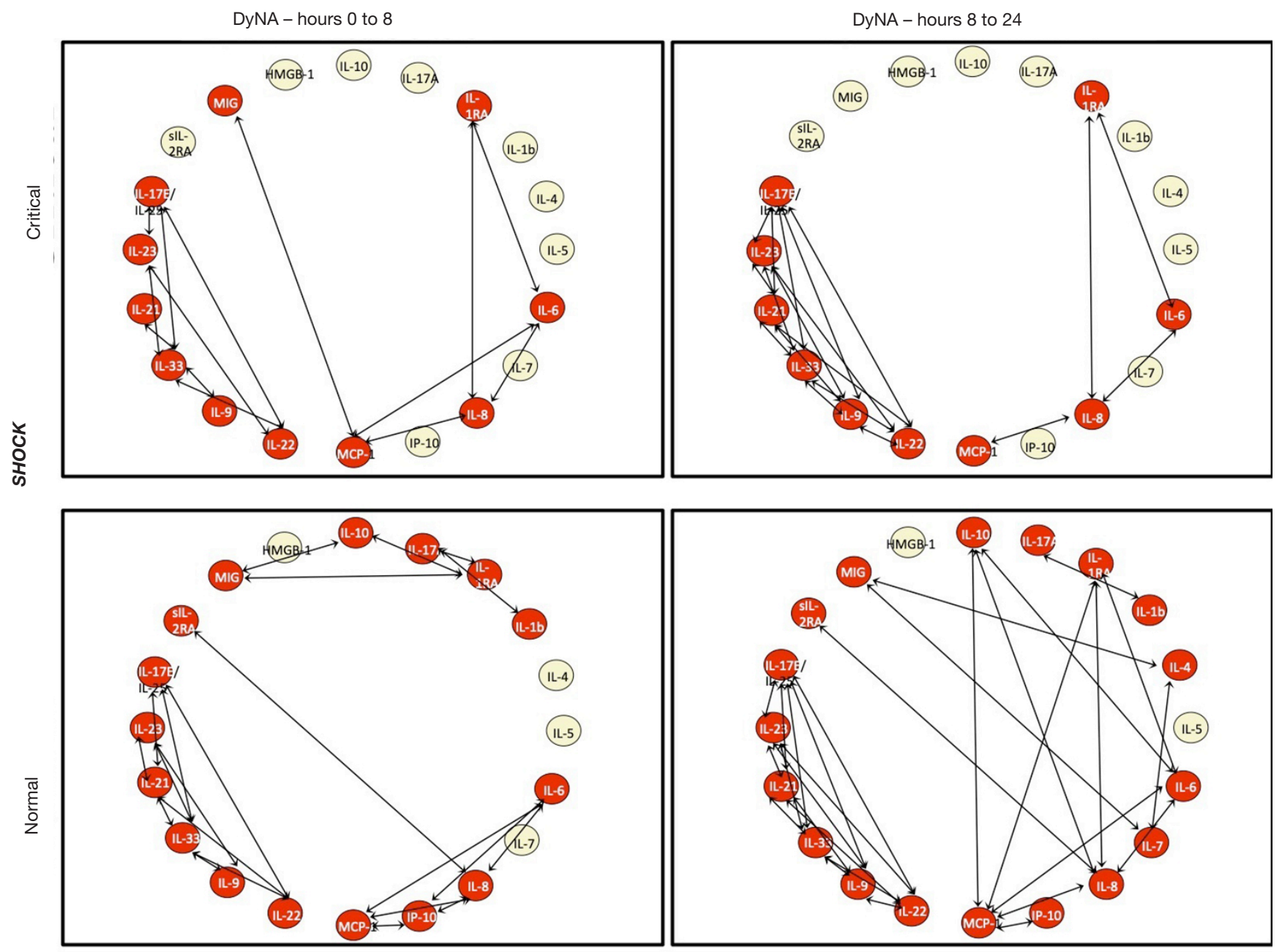

Figure 4 Dynamic network analysis (DyNA) demonstrating inflammatory coordination for the Shock subgroup analysis (matched for age, gender, ISS and admission base deficit). The top row represents CRITICAL patients at two time intervals (0-8 and 8-24 hours), while the bottom row represents normal patient over the same interval. Inflammatory mediators are nodes, with red nodes dynamically involved in interactions, represented by the arrows. DyNa, Dynamic Network Analysis; IL-10, Interleukin 10; IL-6, Interleukin 6; MCP-1, Monocyte Chemoattractant Protein 1; HMGB-1, High Mobility Group Box 1; IL17E/IL-25, Interleukin 17E/Interleukin 25; IL-1RA, Interleukin 1 Receptor Antagonist; MIG, Monokine Induced by Gamma Interferon; IP-10, Interferon $\gamma$-induced Protein 10; IL-8, Interleukin 8; sIL2RA, Soluble Interleukin 2 Receptor $\alpha$; IL-22, Interleukin 22; IL 23, Interleukin 23; IL-33, Interleukin 33; IL-17A, Interleukin 17A; IL-21, Interleukin 21; IL-7, Interleukin 7; IL-9, Interleukin 9; IL 1b, Interleukin 1ß; IL-5, Interleukin 5; IL 4, Interleukin 4.

The inflammatory and coagulation cascades are complex processes which interact with, and influence, one another. These interactions are dynamic and have increasingly been described using computational modeling techniques like Dynamic Bayesian networks (DNB) and Dynamic Network Analysis (DyNa). DyNa is particularly helpful in evaluation of networks over distinct time intervals. The DyNa allows identification of the primary inflammatory mediators (nodes) and their interactions with other biomarkers at various time points, and in specific patient subgroups $(26,27)$.

Our data demonstrated differences in individual immunoactive molecules in the early period following injury. There were significant decreases in immunologic coordination, as reflected in the decreased connectivity seen in the DyNA of CRITICAL patients. Taken together, these findings identify patient-specific differences in the immunologic response to injury that are explicitly associated with a coagulopathic phenotype. 
To further quantify the inflammatory response to trauma, we focused on eight cytokines a priori, due to their established relationships to injury and inflammation or SIRS. Interleukin-6 is produced by T-lymphocytes and macrophages, is a pro-inflammatory mediator and amplifies the inflammatory response $(4,13,16)$. Hepatocytes are also a major source of IL-6 in situations of sterile inflammation, such as injury, and IL-6 drives thrombin production by monocytes (28). IL-6 has variably been associated with infectious complications, MOD, and mortality (17,28-30). Interleukin-8 attracts polymorphonuclear leukocytes and macrophages to wound sites, and has also demonstrated significant elevations in the setting of tissue injury (4). Interleukin-1 cytokines are proinflammatory and contribute to thrombin generation, with elevations seen in disseminated intravascular coagulation (DIC) (14). Interleukin-10, MCP-1, and HMGB1 have all been associated with a pro-inflammatory response and shock $(6,13)$. Interleukin-4 is an anti-inflammatory cytokine and is suppressed following trauma (13). Interleukin-23 (IL-23) has been associated with both pro- and antiinflammatory roles. IL-23 drives Th17 cell differentiation, which likely play a role in the pro-inflammatory response to trauma (18,31).

Initial correlation analysis was performed between the eight potentially significant cytokines and the individual components of the MAR ratio-the R-time and the MA. A significant positive correlation was noted between increasing concentrations of IL-6, IL-8, and MCP-1 and longer R-times. Interpreted one way, increases in these inflammatory mediators is associated with delays in the onset of clot formation. Conversely, a significant negative association was detected between increasing values of IL6, IL-8, IL-10, and MCP-1 and decreasing MA. These increased inflammatory mediators are thus associated with the formation of weaker clot.

Changes in inflammatory mediator concentrations over time were also assessed with linear growth-curve modeling. For this analysis, we used the calculated MAR ratio and controlled for age and ISS. In model development, we considered gender, due to some literature supporting differences in coagulation response by gender, but this was not significant to the model and was discarded. Based on previous work, we used a cut-off MAR ratio of 14.2 with ratios < 14.2 (CRITICAL) associated with worse outcomes (10).

CRITICAL patients had significantly higher values of IL-6, IL-8, IL-10, and MCP-1 at admission than did patients with NORMAL ratios. All cytokine concentrations decreased at a significant rate compared to the admission value. We performed a subgroup analysis in an attempt to isolate the interaction between coagulopathy, as seen in the CRITICAL and NORMAL patients, and the inflammatory response. In order to do this, we used propensity matching to control for patient age, gender, magnitude of injury and, in a further refined match, magnitude of shock at admission as represented by base deficit.

The subgroup analysis demonstrated that CRITICAL patients had significantly different concentrations of key mediators, most noticeable at time of admission. The DyNA also demonstrated that there were significant differences in the coordination of the immune response between groups. CRITICAL patients also displayed a significantly less coordinated early inflammatory response to injury, with fewer involved inflammatory mediators, most notable in the earlier time-point. In comparison, the NORMAL patients had a much more coordinated response, as demonstrated by the greater interaction between mediators at early time points and 24 hours.

Patients with low ratios are unique and the CRITICAL MAR is only the tip of an iceberg. CRITICAL and NORMAL patients appear very similar at the time of admission, in terms of gender, age and injury severity. Clinical gestalt is therefore not enough to identify this phenotype. The low MAR ratio in CRITICAL patients identifies a malfunction of the coagulation system that may initially be subtle, yet indicative of a progressive traumatic coagulopathy. Even when normalized for injury severity and hemorrhage, these same patients demonstrated abnormal and poorly coordinated inflammatory responses. The importance of the MAR ratio is to indicate the profound uncoupling of multiple systems, at the molecular level, in response to severe injury.

The study does have limitations, one of which is size. Overall mortality was low in both groups despite a high burden of injury. No significant difference in mortality, or hospital and intensive care unit length of stay, was demonstrated between these cohorts. However, the purpose of this study was to assess coagulopathy and the inflammatory response following major injury and, therefore, the study was not powered to investigate differences in mortality. Prior work on the MAR ratio does demonstrate a mortality difference (10). Additionally, subgroup analysis resulted in small cohort sizes used in 
DyNa for both INJURY and SHOCK groups. Though the analysis controlled for important patient variables and did demonstrate significant differences in immunologic coordination, the small groups may have introduced an unintentional bias.

Additionally, the population of this study primarily suffered a blunt mechanism of injury. Patients with severe blunt and penetrating injuries are physiologically different, so results of this study cannot be assumed applicable for penetrating trauma patients without further investigation. Finally, the relationship between DyNA and the MAR ratio was evaluated using a dichotomous cutoff, with the CRITICAL and NORMAL thresholds derived from our previous work. There may be more meaningful physiologic relationships that exist between other MAR values, on a continuous scale, and trauma-relevant phenotypes. However, we chose thresholds for this analysis based on our initial work and our data demonstrate that the initial MAR ratio serves as an effective biomarker to anticipate higher volume hemorrhage and immunologic dysfunction after injury.

In conclusion, the MAR ratio is one piece of a much larger puzzle representing the complicated response to severe injury. Rather than producing a predictable inflammatory progression, blunt injury initiates a 'storm' of inflammatory mediators both innate and adaptive. These participate in a complex interplay with various systems, including coagulation.

Patients who progress to TIC demonstrate a significant interaction between dysfunctional clot formation and alterations in inflammatory mediators. Differences in initial immunologic response in TIC patients were affected by the magnitude of injury and hemorrhage, but these differences persisted in sub-cohorts of patients normalized for ISS and base deficit, indicating that patient-specific differences in response to injury may affect TIC. In effect, injury merely initiates a complex interplay between clot formation and inflammation that is expressed as one phenotype of TIC. The MAR ratio is the earliest warning signal of one such pathologic phenotype.

\section{Acknowledgments}

The authors would like to thank Dr. John Holcomb for his review and recommendations regarding restructuring this scientific paper. Parts of this study were presented at the $76^{\text {th }}$ annual meeting of the American Association for the Surgery of Trauma (Baltimore, MD, 2017) and the $31^{\text {st }}$ annual meeting of the Eastern Association for the Surgery of Trauma (Orlando, FL, 2018).

\section{Footnote}

Reporting Checklist: The authors have completed the STROBE reporting checklist. Available at http://dx.doi. org/10.21037/atm-20-3651

Data Sharing Statement: Available at http://dx.doi. org/10.21037/atm-20-3651

Peer Review File: Available at http://dx.doi.org/10.21037/ atm-20-3651

Conflicts of Interest: All authors have completed the ICMJE uniform disclosure form (available at http://dx.doi. org/10.21037/atm-20-3651). YV serves as an unpaid editorial board member of Annals of Translational Medicine from Mar 2019 to Feb 2021. Dr. YV reports grants from Indiana University internal funds, during the conduct of the study; other from Immunetrics, Inc., outside the submitted work. Dr. RAC reports grants from NIH, during the conduct of the study. Dr. TOM reports personal fees from Innomed, outside the submitted work. The other authors have no conflicts of interest to declare.

Etbical Statement: The authors are accountable for all aspects of the work in ensuring that questions related to the accuracy or integrity of any part of the work are appropriately investigated and resolved. The study was conducted in accordance with the Declaration of Helsinki (as revised in 2013). The study was approved Institutional Review Board of Indiana University School of Medicine (number 1411863767) and informed consent was taken from all individual participants.

Open Access Statement: This is an Open Access article distributed in accordance with the Creative Commons Attribution-NonCommercial-NoDerivs 4.0 International License (CC BY-NC-ND 4.0), which permits the noncommercial replication and distribution of the article with the strict proviso that no changes or edits are made and the original work is properly cited (including links to both the formal publication through the relevant DOI and the license). See: https://creativecommons.org/licenses/by-nc-nd/4.0/.

\section{References}

1. Inflammation and the Host Response to Injury. A Multi- 
Disciplinary Research Program. Inflammation and Host Response to Injury. Available online: http://www. gluegrant.org/

2. MacLeod JBA. Trauma and coagulopathy. A new paradigm to consider. Arch Surg 2008;143:797-801.

3. Christie SA, Kornblith LZ, Howard BM, et al. Characterization of distinct coagulopathic phenotypes in injury: Pathway-specific drivers and implications for individualized treatments. J Trauma Acute Care Surg 2017;82:1055-62.

4. Reikerås $\mathrm{O}$, Borgen P. Activation of markers of inflammation, coagulation and fibrinolysis in musculoskeletal trauma. PLoS One 2014;9:e107881.

5. Bronkhorst MWGA, Patka P, Van Lieshout EMM. Effects in sequence variations in innate immune response genes on infectious outcome in trauma patients: A comprehensive review. Shock 2015;44:390-6.

6. Sauaia A, Moore FA, Moore EE. Postinjury inflammation and organ dysfunction. Crit Care Clin 2017;33:167-91.

7. Xiao W, Mindrinos MN, Seok J, et al. A genomic storm in critically injured humans. J Exp Med 2011;208:2581-90.

8. Bogner V, Keil L, Kanz KG, et al. Very early posttraumatic serum alterations are significantly associated to initial massive RBC substitution, injury severity, multiple organ failure and adverse clinical outcome in multiple injured patients. Eur J Med Res 2009;14:284-91.

9. Petäjä J. Inflammation and Coagulation. An Overview Thromb Res 2011;127:S34-7.

10. Savage SA, Zarzaur BL, Pohlman TH, et al. Clot dynamics and mortality: The MA-R ratio. J Trauma Acute Care Surg 2017;83:628-34.

11. Savage SA, Sumislawski JJ, Zarzaur BL, et al. The new metric to define large-volume hemorrhage: results of a prospective study of the critical administration threshold. J Trauma Acute Care Surg 2015;78:224-9; discussion 229-30.

12. Namas R, Mi Q, Namas R, et al. Insights into the role of chemokines, damage-associated molecular patterns, and lymphocyte-derived mediators from computational models of trauma-induced inflammation. Antioxid Redox Signal 2015;23:1370-87.

13. Volpin G, Cohen M, Assaf M, et al. Cytokine levels (IL4, IL-6, IL-8 and TGF-beta) as potential biomarkers of systemic inflammatory response in trauma patients. Int Orthop 2014;38:1303-9.

14. Gando S, Kameue T, Matsuda N, et al. Combined activation of coagulation inflammation has an important role in multiple organ dysfunction and poor outcome after severe trauma. Thromb Haemost 2002;88:943-9.

15. Stutz CM, O'Rear LD, O’Neill KR, et al. Coagulopathies in orthopedics: Links to inflammation and the potential of individualizing treatment strategies. J Orthop Trauma 2013;27:236-41.

16. Sousa A, Raposo F, Fonseca S, et al. Measurement of cytokines and adhesion molecules in the first 72 hours after severe trauma: Association with severity and outcome. Dis Markers 2015;2015:747036.

17. Biffl WL, Moore EE, Moore FA. Interleukin 6 in the injured patient marker of injury or mediator of inflammation? Ann Surg 1996;224:647-64.

18. Torrance HDT, Vivian ME, Brohi K, et al. Changes in gene expression following trauma are related to the age of transfused packed red blood cells. J Trauma Acute Care Surg 2015;78:535-42.

19. Cohen MJ, Christie SA. Coagulopathy of trauma. Crit Care Clin 2017;33:101-18.

20. Chen R, Herskovits EH, the Alzheimer's Disease Neuroimaging Initiative. Predictive structural dynamic network analysis. J Neurosci Methods 2015;245:58-63.

21. Chen R, Resnick SM, Davatzikos C, et al. Dynamic Bayesian network modeling for longitudinal brain morphometry. Neuroimage 2012;59:2330-8.

22. Dzieciatkowska M, D'Alessandro A, Moore EE, et al. Lymph is not a plasma ultrafiltrate: A proteomic analysis of injured patients. Shock 2014;42:485-98.

23. Sauaia A, Moore EE, Johnson JL, et al. Temporal trends of postinjury multiple-organ failure: still resource intensive, morbid and lethal. J Trauma Acute Care Surg 2014;76:58292, discussion 592-3.

24. Dewar DC, Tarrant SM, King KL, et al. Changes in the epidemiology and prediction of multiple-organ failure after injury. J Trauma Acute Care Surg 2013;74:774-9.

25. Cook R, Cook D, Tilley J, et al. Multiple organ dysfunction: baseline and serial component scores. Crit Care Med 2001;29:2046-50.

26. Zamora R, Vodovotz Y, Mi Q, et al. Data-driven modeling for precision medicine in pediatric acute liver failure. Mol Med 2017;22:821-9.

27. Mi Q, Constantine G, Ziraldo C, et al. A dynamic view of trauma/hemorrhage-induced inflammation in mice: principal drivers and networks. PLoS One 2011;6:e19424.

28. Norris CA, He M, Kang Li, et al. Synthesis of IL-6 by hepatocytes is a normal response to common hepatic stimuli. PLoS One 2014;9:e96053.

29. Ertel W, Faist E, Nestle C, et al. Kinetics of interleukin-2 and interleukin-6 synthesis following major mechanical 
trauma. J Surg Res 1990;48:622-8.

30. Svoboda P, Kantorova I, Ochmann J. Dynamics of interleukin 1, 2, and 6 and tumor necrosis factor alpha in multiple trauma patients. J Trauma 1994;36:336-40.

Cite this article as: Savage SA, Zarzaur BL, Gaski GE, McCarroll T, Zamora R, Namas RA, Vodovotz Y, Callcut RA, Billiar TR, McKinley TO. Insights into the association between coagulopathy and inflammation: abnormal clot mechanics are a warning of immunologic dysregulation following major injury. Ann Transl Med 2020;8(23):1576. doi: 10.21037/atm-20-3651
31. Abboud A, Namas RA, Ramadan M, et al. Computational analysis supports an early, type 17 cell-associated divergence of blunt trauma survival and mortality. Crit Care Med 2016;44:e1074-81. 